\title{
Metabolic Engineering of Extremophilic Bacterium Deinococcus radiodurans for the Production of the Novel Carotenoid Deinoxanthin
}

\author{
Sun-Wook Jeong ${ }^{1}{ }^{(D)}$, Jun-Ho Kim ${ }^{2}$, Ji-Woong $\mathrm{Kim}^{2}$, Chae Yeon Kim ${ }^{2}$, Su Young Kim ${ }^{2}$ and Yong Jun Choi ${ }^{1, *(D)}$ \\ 1 School of Environmental Engineering, University of Seoul, Seoul 02504, Korea; jeongsunwook@gmail.com \\ 2 Material Sciences Research Institute, LABIO Co., Ltd., Seoul 08501, Korea; msri06@labio.kr (J.-H.K.); \\ msri12@labio.kr (J.-W.K.); msri02@labio.kr (C.Y.K.); msri01@labio.kr (S.Y.K.) \\ * Correspondence: yongjun2165@uos.ac.kr; Tel.: +82-02-6490-2873; Fax: +82-02-6490-2859
}

Citation: Jeong, S.-W.; Kim, J.-H.; Kim, J.-W.; Kim, C.Y.; Kim, S.Y.; Choi, Y.J. Metabolic Engineering of

Extremophilic Bacterium Deinococcus radiodurans for the Production of the Novel Carotenoid Deinoxanthin.

Microorganisms 2021, 9, 44.

https://doi.org/10.3390/

microorganisms 9010044

Received: 27 November 2020

Accepted: 23 December 2020

Published: 25 December 2020

Publisher's Note: MDPI stays neutral with regard to jurisdictional claims in published maps and institutional affiliations.

Copyright: (c) 2020 by the authors. Licensee MDPI, Basel, Switzerland. This article is an open access article distributed under the terms and conditions of the Creative Commons Attribution (CC BY) license (https: / / creativecommons.org/ licenses/by/4.0/).

\begin{abstract}
Deinoxanthin, a xanthophyll derived from Deinococcus species, is a unique organic compound that provides greater antioxidant effects compared to other carotenoids due to its superior scavenging activity against singlet oxygen and hydrogen peroxide. Therefore, it has attracted significant attention as a next-generation organic compound that has great potential as a natural ingredient in a food supplements. Although the microbial identification of deinoxanthin has been identified, mass production has not yet been achieved. Here, we report, for the first time, the development of an engineered extremophilic microorganism, Deinococcus radiodurans strain R1, that is capable of producing deinoxanthin through rational metabolic engineering and process optimization. The genes $c r t B$ and $d x$ s were first introduced into the genome to reinforce the metabolic flux towards deinoxanthin. The optimal temperature was then identified through a comparative analysis of the mRNA expression of the two genes, while the carbon source was further optimized to increase deinoxanthin production. The final engineered $D$. radiodurans strain $R 1$ was able to produce $394 \pm 17.6 \mathrm{mg} / \mathrm{L}(102 \pm 11.1 \mathrm{mg} / \mathrm{g}$ DCW) of deinoxanthin with a yield of $40.4 \pm 1.2 \mathrm{mg} / \mathrm{g}$ sucrose and a productivity of $8.4 \pm 0.2 \mathrm{mg} / \mathrm{L} / \mathrm{h}$ from $10 \mathrm{~g} / \mathrm{L}$ of sucrose. The final engineered strain and the strategies developed in the present study can act as the foundation for the industrial application of extremophilic microorganisms.
\end{abstract}

Keywords: Deinococcus radiodurans; metabolic engineering; deinoxanthin; xanthophylls; antioxidation

\section{Introduction}

Xanthophylls are oxygenated carotenoids that have received significant interest as an ingredient in natural foods due to their association with beauty and health. In particular, xanthophylls have distinctive structural features such as terminal hydroxy groups, ketones, aldehyde, and epoxy groups that provide superior radical scavenging activity compared with carotenes such as $\beta$-carotene and lycopene [1-3]. Over the last few decades, significant research progress has been made on the microbial production of xanthophylls using microalgae, yeast, and other microorganisms. For example, the biosynthetic pathway for astaxanthin, zeaxanthin, and lutein, which are widely used as dietary supplements, has been discovered in various microorganisms [4-6]. Recently, (3R)-saproxanthin and (3R,2'S)myxol, which enhance lipid peroxidation activity compared with $\beta$-carotene, were isolated from the marine bacterial strains 04OKA-13-27 and YM6-073, respectively [7]. Another study also reported the isolation of siphonaxanthin, which is considered a promising anti-cancer compound, from green algae within the order Siphonales [8].

Recently, extremophilic microorganisms have been recognized as a promising bioresource for the production of various xanthophylls. This is because they have evolved to produce their own xanthophylls that are involved in multiple cellular functions essential for their survival in extreme habitats. For example, $2^{\prime}$-isopentenylsaproxanthin was first discovered in the alkaliphilic bacterium Jejuia pallidilutea strain 11shimoA1 [9]. The levels 
of $2^{\prime}$-isopentenylsaproxanthin have been found to increase under high alkaline conditions ( $\mathrm{pH}$ 9.2), suggesting that 2 -isopentenylsaproxanthin plays a crucial role in resisting extreme conditions. Another study also reported the isolation of thermozeaxanthin from the thermophilic bacterium Thermus filiformis, which is responsible for adaption to extremely high temperatures by stabilizing the cellular membrane [10].

Deinococcus radiodurans strain R1 (reclassified from Micrococcus radiodurans) is a radiationresistant extremophilic microorganism that has great potential as a microbial host for the production of novel carotenoids [11]. Recently, metabolically engineered D. radiodurans R1 capable of producing phytoene, a colorless carotenoid, was reported for the first time [12]. The high microbial production of lycopene has also been successfully achieved via systematic metabolic engineering approaches in combination with an optimized abiotic environment, such as the presence of UV-C irradiation and conditional nutrient supplementation $[13,14]$. In particular, deinoxanthin ((2R)-2, $1^{\prime}$-dihydroxy-3' ${ }^{\prime} 4^{\prime}$-didehydro- $1^{\prime}, 2^{\prime}$-dihydro$\beta, \Psi$-caroten-4-one) is a unique xanthophyll that can only be synthesized by Deinococcus microorganisms and is possibly associated with the ability to survive under extremely high irradiation $[11,15,16]$. It has also demonstrated a higher scavenging activity against singlet oxygen and hydrogen peroxide $\left(\mathrm{H}_{2} \mathrm{O}_{2}\right)$ than other xanthophyll carotenoids such as lutein and zeaxanthin [17-19]. In a previous comparative study on various xanthophyll structures, deinoxanthin was shown to possess a unique structural feature consisting of extended conjugated double-bonds, a conjugated keto-group, a C-1' hydroxyl group, and an additional C-2 hydroxyl group on the $\beta$-ring end, which may be responsible for its superior antioxidation activity [16]. Despite the significant potential of deinoxanthin as a beneficial natural compound, only molecular-level research, including the optimization of culture conditions and carbon sources, has been conducted to date [15,20-24], and no studies have been reported on its mass production via systematic metabolic engineering. Thus, the production of deinoxanthin via systematic metabolic engineering of $D$. radiodurans $\mathrm{R} 1$ remains an important goal. In the present study, we report, for the first time, the engineering of the carotenoid biosynthetic pathway in D. radiodurans $\mathrm{R} 1$ to produce large amounts of deinoxanthin.

\section{Materials and Methods}

\subsection{Strains, Plasmids, and Culture Medium}

The strains and plasmids used in the present study are listed in Table 1. Deinococcus radiodurans $\mathrm{R} 1$ (ATCC13939) and its derivatives were cultivated in TGY media (0.5\% trytone, $0.1 \%$ glucose, and $0.3 \%$ yeast extract). Batch culturing for the production of deinoxanthin was conducted in modified TGY medium (per liter, $\mathrm{pH} 7.0$ ) containing $5 \mathrm{~g} / \mathrm{L}$ tryptone, $5 \mathrm{~g} / \mathrm{L}$ yeast extract, $10 \mathrm{~g} / \mathrm{L}$ glucose, $0.5 \mathrm{~g} / \mathrm{L} \mathrm{MgSO}_{4} \cdot 7 \mathrm{H}_{2} \mathrm{O}$, and $1 \mathrm{mg} / \mathrm{L} \mathrm{MnCl}_{2}$. Escherichia coli $\mathrm{DH} 5 \alpha$ was used as the host strain for the construction of recombinant plasmids and cultured at $37^{\circ} \mathrm{C}$ in Luria-Bertani (LB) medium. When necessary, antibiotics were added at a final concentration of $100 \mu \mathrm{g} / \mathrm{mL}$ ampicillin for E. coli, and $3 \mu \mathrm{g} / \mathrm{mL}$ chloramphenicol and $25 \mu \mathrm{g} / \mathrm{mL}$ kanamycin for $D$. radiodurans.

\subsection{DNA Manipulation and Plasmid Construction}

The primer sets used for plasmid construction are listed in Table S1. All recombinant plasmids and PCR products were purified using the MegaQuick-Spin plus fragment DNA purification kit (Intron Biotechnology, Seongnam, Korea) and the NucleoSpin kit (Macherey-Nagel GmbH \& Co., Dueren, Germany). The recombinant plasmids pAM41, pAM73, and pAM104 were obtained from a previous study (Table 1) [12]. The pAM104 plasmid was directly transformed into D. radiodurans following a standard protocol [12], and the transformants were screened on $2 \times$ TGY-chloramphencol agar plates. The resulting $D$. radiodurans strain expressing $d x s$ and $c r t B$ was designated as DX1. 
Table 1. Strains and plasmids used in the present study.

\begin{tabular}{|c|c|c|}
\hline & Description & Reference \\
\hline Strains & & \\
\hline D. radiodurans $\mathrm{R} 1$ & Wild type (ATCC13939) & ATCC \\
\hline D. radiodurans DX1 & Wild-type harboring pAM104 plasmid & This study \\
\hline D. radiodurans DX2 & Integration of $d x s$ and $c r t B$ genes with the loxP scar sequence into the $D$. radiodurans $\mathrm{R} 1$ chromosome (CP1) & This study \\
\hline $\begin{array}{l}\text { E. coli } \mathrm{DH} 5 \alpha \\
\text { Plasmids }\end{array}$ & Host for recombinant plasmid construction & Lab stock \\
\hline pTOP Blunt V2 & TA cloning vector & Enzynomics \\
\hline pRADZ3 & E. coli-D. radiodurans shuttle vector carrying the groE promoter & [25] \\
\hline pAM1 & Derivative of pKatAPH3 containing a lox $66-\mathrm{km}^{r}$-lox71 cassette & [25] \\
\hline pAM2 & Derivative of p13840 containing $\mathrm{P}_{\text {groE }}-\mathrm{cre}^{-} \mathrm{P}_{\text {groE }}-\mathrm{cm}^{r}$ & [25] \\
\hline pAM41 & Derivative of pRADZ3 containing $\operatorname{crt} B$ & [12] \\
\hline pAM73 & Derivative of pRADZ3 containing $d x s$ & [12] \\
\hline pAM104 & Derivative of pRADZ3 containing $d x s$ and $c r t B$ & [12] \\
\hline Pdrc6-crtB & $\begin{array}{c}\text { pTOP Blunt V2 containing two homology arms (the partial sequences of } d r C 0006 \text { and } d r c 0007 \text { ) } \\
\text { and } \mathrm{P}_{\text {groE }}-\mathrm{crtB}-\operatorname{lox} 66-\mathrm{km}^{r}-\mathrm{lox} 71\end{array}$ & This study \\
\hline Pdrc4-dxs & $\begin{array}{l}\text { pAM1 containing two homology arms (the partial sequences of } d r C 0004 \text { and } d r c 0005 \text { ) } \\
\text { and } \mathrm{P}_{g r o E^{-}}-d x s-l o x 66-\mathrm{km}^{r}-l o x 71\end{array}$ & This study \\
\hline
\end{tabular}

The endogenous genes $c r t B$ and $d x$ s under the groE promoter were integrated into the $D$. radiodurans chromosome $\mathrm{CP} 1$ between the $d r \mathrm{C} 0004$ and $d r c 0007$ regions using a Cre-loxP system [25]. The gene integration process is depicted in Figure S1. To integrate $c r t B$ into the $D$. radiodurans genome, approximately $1 \mathrm{~kb}$ of DNA fragments containing partial sequences of $d r C 0006$ and $d r C 0007$ was amplified via PCR using appropriate primer sets. The resulting PCR fragments were assembled using fusion PCR and ligated with pTOP Blunt V2 plasmid (Enzynomics) (designated as pdrc6). The DNA fragment containing $c r t B$ and the groE promoter was amplified using groE-F1/crtB-R1 primers and the pAM41 plasmid as a DNA template. The resulting PCR product was digested with KpnI/EcoRV and ligated into pAM1. The DNA fragment containing the groE promoter, $\operatorname{crt} B$, and the kanamycin resistance cassette flanked with two loxP sequences was amplified using a groE-F2/crtB-R2 primer set. The resulting PCR product was ligated into the pdrc6 plasmid at the StuI site (referred to as pdrc6-crtB, Table 1). An integrative plasmid for the insertion of $d x$ s was also constructed. Approximately $1 \mathrm{~kb}$ of upstream/downstream DNA fragments containing $d r C 0004$ and $d r c 0005$ was amplified using drc04-1/drc04-2 and drc05-1/drc05-2 primer sets, respectively. The PCR fragments were ligated into the pAM1 plasmid at the KpnI/Pst I and BamHI/SphI sites (designated as pdrc4). The DNA fragment containing $d x$ s and the groE promoter was amplified using $\mathrm{dxs}-\mathrm{F} 1 / \mathrm{dxs}-\mathrm{R} 1$ primers and the pAM73 plasmid as a DNA template. The resulting PCR product was digested with PstI/EcoRV and ligated with pdrc4 in the same way as for the restriction enzyme sites (referred to as pdrc4-dxs, Table 1). The integrated plasmids pdrc4-dxs and pdrc6-crtB were sequentially transformed into $D$. radiodurans, and the resulting transformants were screened using a $2 \times$ TGYkanamycin agar plate. To remove the kanamycin resistance gene from the $D$. radiodurans genome, pAM2, which expresses Cre recombinase, was transformed and completely cured in $D$. radiodurans following a previously described method [25]. Gene integration and the selective removal of markers in the $d x s$ - and $c r t B$-integrated $D$. radiodurans mutants were confirmed using plating assays, diagnostic PCR using appropriate primer sets, and DNA sequencing (Figure S2). This final strain was designated as D. radiodurans DX2 and used for deinoxanthin production.

\subsection{Shake Flask Cultivation}

To examine deinoxanthin production in the engineered $D$. radiodurans strains, fresh colonies were inoculated into $3 \mathrm{~mL}$ of TGY medium and cultivated overnight $(16-20 \mathrm{~h})$ at $30{ }^{\circ} \mathrm{C}$ and $200 \mathrm{rpm}$ to produce an $\mathrm{OD}_{600}$ of 1.8-2.1. Seed cultures (1\%) were then transferred to a $250 \mathrm{~mL}$ baffled flask with $50 \mathrm{~mL}$ of modified TGY medium and cultivated at $30{ }^{\circ} \mathrm{C}$ and $200 \mathrm{rpm}$ for $48 \mathrm{~h}$. To examine the effects of temperature and the carbon source on cell growth and deinoxanthin production, the D. radiodurans strain DX2 was initially cultivated 
in $50 \mathrm{~mL}$ of modified TGY medium at $30{ }^{\circ} \mathrm{C}$ for $24 \mathrm{~h}$ and then grown at 30,32 , or $37^{\circ} \mathrm{C}$ for $48 \mathrm{~h}$ after the change in temperature. Glucose, sucrose, and fructose were used as a carbon source $(10 \mathrm{~g} / \mathrm{L})$ in modified TY medium ( $\mathrm{pH} 7.0)$. Cell growth was monitored by measuring the absorbance at $600 \mathrm{~nm}\left(\mathrm{OD}_{600}\right)$ using a microplate reader (Biotek, Winooski, VT, USA). Cultured broth $(10 \mathrm{~mL})$ was harvested by centrifugation at $4000 \mathrm{rpm}$ for $20 \mathrm{~min}$ and washed twice with deionized water (DW). The obtained pellets were freeze-dried and the weight of the dried cells was measured before carotenoid extraction.

\subsection{Carotenoid Extraction and Analytical Methods}

Total carotenoids from the pellets were extracted using $1 \mathrm{~mL}$ of methanol and sonication for $1 \mathrm{~min}$, which was repeated twice. To produce standard deinoxanthin, $1 \mathrm{~L}$ of wild-type $D$. radiodurans cells grown for $48 \mathrm{~h}$ at $30{ }^{\circ} \mathrm{C}$ was harvested at $4000 \mathrm{rpm}$ for $30 \mathrm{~min}$. After washing twice with DW, deinoxanthin was extracted from the pellets with $50 \mathrm{~mL}$ of methanol. The deinoxanthin extracts were concentrated using a rotary evaporator (IKA, Seoul, Korea) at $55^{\circ} \mathrm{C}$ and $70 \mathrm{rpm}$ and completely lyophilized using a freeze dryer (Operon Co., Ltd., Gimpo, Korea). A deinoxanthin calibration curve (5 to $200 \mathrm{mg} / \mathrm{L}$ ) was constructed to quantify the deinoxanthin levels.

Carotenoid analysis was conducted using previously described methods [12,24]. The deinoxanthin was analyzed using high-performance liquid chromatography (HPLC, Agilent 1260 InfinityII, Agilent Corp., Santa Clara, CA, USA) equipped with a Zorbax Eclipse XDB-C18 column (4.6 × 150 mm; Agilent Corp., Santa Clara, CA, USA) and a UV/VIS detector at $480 \mathrm{~nm}$. A mixture of acetonitrile, methanol, and isopropanol (40:50:10,v/v/v) was used as the mobile phase with a flow rate of $0.8 \mathrm{~mL} \mathrm{~min}^{-1}$ at $40{ }^{\circ} \mathrm{C}$. The concentration of the carbon source in the culture media was determined using HPLC equipped with a refractive index detector. Separation was conducted with a MetaCarb $87 \mathrm{H}$ column $\left(4.6 \times 250 \mathrm{~mm}\right.$; Agilent Corp., Santa Clara, CA, USA) at $60{ }^{\circ} \mathrm{C}$. As the mobile phase, $0.005 \mathrm{~N}$ $\mathrm{H}_{2} \mathrm{SO}_{4}$ was used at a flow rate of $0.5 \mathrm{~mL} \mathrm{~min}^{-1}$. LC-MS analysis was performed using an LC/MS detection system (Agilent 6120 Quadrupole LC/MS, Agilent Corp., Santa Clara, CA, USA) to determine the molecular mass of deinoxanthin from the crude extracts. The LC/MS conditions were as follows: a drying gas temperature of $350{ }^{\circ} \mathrm{C}$, a drying gas flow rate of $10 \mathrm{~L} \mathrm{~min}^{-1}$, a capillary voltage of $3.5 \mathrm{kV}$, and a nebulizer pressure of $40 \mathrm{psi}_{\mathrm{g}}$.

\subsection{Real-Time Quantitative PCR (RT-qPCR)}

Total RNA was extracted from $1 \mathrm{~mL}$ of $D$. radiodurans cells with RiboEX reagent (GeneAll, Seoul, Korea) according to the manufacturer's instructions. Cell disruption was instigated by adding $500 \mu \mathrm{L}$ of $0.1-\mathrm{mm}$ diameter silica beads (Biospec, Bartlesville, OK, USA). DNA contaminants were completely removed from the crude lysates using DNase I (Takara, Tokyo, Japan) treatment and purified using an RNeasy Mini Kit (Qiagen, Hilden, Germany). For real-time quantitative PCR analysis (RT-qPCR), cDNA was synthesized from $1 \mu \mathrm{g}$ of total RNA using a SuperiorScript III cDNA synthesis kit (Enzynomics, Daejeon, Korea) following the manufacturer's instructions. RT-qPCR amplification was conducted using the RbTaq Fast qPCR 2X premix (Enzynomics, Daejeon, Korea) on a Bio-Rad CFX Real-Time System (Bio-Rad, Hercules, California, USA). PCR reactions were conducted using the following process: one cycle of $95^{\circ} \mathrm{C}$ for $3 \mathrm{~min}$, followed by 40 cycles of $95{ }^{\circ} \mathrm{C}$ for $10 \mathrm{~s}$ and $60{ }^{\circ} \mathrm{C}$ for $30 \mathrm{~s}$. The gene $d r 1343$, which encodes glyceraldehyde3-phosphate dehydrogenase (gap), was chosen as an internal control to normalize the transcriptional expression of the tested target genes. Approximately $100 \mathrm{bp}$ of unique sequences from the genes encoding gap (dr1343), groE (dr0606), crtB (dr0862), and $d x s(d r 1475)$ was amplified using the following primers: dr1343F $/ \mathrm{R}\left(5^{\prime}-\right.$ caacgacctgaccgacaacc- $3^{\prime}, 5^{\prime}-$ ggctgctttcgtcgtactcc- $\left.3^{\prime}\right)$, dr0606F/R (5' -gtcaaggaaggcgacaccgt- $3^{\prime}, 5^{\prime}$-tcgacaatggcgagcaggtc$\left.3^{\prime}\right)$, dr0862F/R (5'-caggccgtattcgtcgagca- $3^{\prime}, 5^{\prime}$-aactcggtcaggcgatgcag $\left.3^{\prime}\right)$, and dr1475F/R (5'-ctgcgcgggatgctcaagta-3', $5^{\prime}$-atttcaggtccggccacgtc $\left.3^{\prime}\right)$. 


\subsection{DPPH Assays}

The antioxidant activity of the carotenoids was determined using 2, 2-diphenyl-1picryl-hydrazyl (DPPH, Alfa Aesar, Tewskbury, MA, USA) free radical assays as previously described [26]. Briefly, $100 \mu \mathrm{L}$ of DPPH solution $(0.2 \mathrm{mM})$ in methanol was added to $100 \mu \mathrm{L}$ of different concentrations of carotenoid solutions and incubated for $30 \mathrm{~min}$ at room temperature in the dark. After the reaction, the absorbance of the samples was measured at $517 \mathrm{~nm}$ using a microplate reader (Biotek, Winooski, VT, USA). The following standard carotenoids were used: phytoene (TRC, North York, ON, Canada), beta-carotene (Sigma-Aldrich, St. Luis, MO, USA), lycopene (Sigma-Aldrich, St. Luis, MO, USA), and astaxanthin (Cayman, MI, USA). Ascorbic acid (TCI, Tokyo, Japan) was used as a positive control in the DPPH assays. Radical scavenging activity was calculated as a percentage of DPPH discoloration using Equation (1):

DPPH scavenging activity $(\%)=[$ Acontrol $-($ Asample - Asample blank $) /$ Acontrol $] \times 100$

where $\mathrm{A}_{\text {contol }}, \mathrm{A}_{\text {sample, }}$ and $\mathrm{A}_{\text {sample blank }}$ are the absorbance of the DPPH solution, the carotenoid solution with DPPH, and the carotenoid solution without DPPH, respectively.

\section{Results}

\subsection{Construction of a Base Strain via the Genome-Based Overexpression of Rate-Limiting Steps}

Previous research has identified the deinoxanthin biosynthetic pathway in Deinococcus strains via the comparative functional analysis of putative carotenoid genes [16,20-23]. According to a previous report, deinoxanthin biosynthesis is initiated through the methylerythritol 4-phosphate (MEP) pathway, and the carotenoid $\gamma$-carotene is utilized as a core backbone at the end of the synthesis process. During this process, unique structural features that lead to superior antioxidant performance are created via enzymatic modifications such as hydroxylation, desaturation, and ketolation mediated by 1,2-hydratase $(\mathrm{CruF})$, carotenoid $3^{\prime}, 4^{\prime}$ - desaturase $(\mathrm{CrtD})$, carotenoid ketolase $(\mathrm{CrtO})$, and carotene $2-\beta$ hydroxlyase (DR2473) (Figure S1) [22].

Previous studies have reported that $d x s$, which encodes 1-deoxy-D-xylulose-5-phosphate synthase, and $c r t B$, which encodes phytoene synthase, are rate-limiting steps in the carotenoid biosynthetic pathway in $D$. radiodurans. Thus, metabolically engineered $D$. radiodurans strains that overexpress both genes have been used as the base strain for the production of various carotenoids $[12,13]$. Because wild-type D. radiodurans also contains the deinoxanthin biosynthetic pathway, $d x s$ and $c r t B$ were overexpressed via plasmid-based overexpression to produce a deinoxanthin-overproducing strain. The resulting strain (DX1) produced $176.1 \pm 4.6 \mathrm{mg} / \mathrm{L}$ of deinoxanthin, twice as much as that obtained from the wild-type strain $(88.3 \pm 3.2 \mathrm{mg} / \mathrm{L})$ (Figure $1 \mathrm{~A})$. This demonstrates that the overexpression of $d x s$ and $c r t B$ also occurred during deinoxanthin production in the $D$. radiodurans strain. However, the cell growth of the DX1 strain $\left(\mathrm{OD}_{600}\right.$ of $\left.12.5 \pm 1.2\right)$ was $39 \%$ lower at the end of culturing than the wild-type $\left(\mathrm{OD}_{600}\right.$ of $\left.20.1 \pm 0.4\right)$ (Figure $\left.1 \mathrm{~B}\right)$. This is probably due to the severe metabolic burden resulting from plasmid-based overexpression [17]. Thus, a $D$. radiodurans strain in which $d x s$ and $c r t B$, regulated by the groE promoter $\left(\mathrm{P}_{\text {groE }}\right)$, were inserted into the genome was constructed using a plasmid-free Cre-loxP system (Figure 1C,D) [25]. As expected, the cellular growth of the resulting strain (DX2) was recovered while maintaining high deinoxanthin production $(177.29 \pm 8.4 \mathrm{mg} / \mathrm{L})$ (Figure 1B). Therefore, the DX2 strain was selected as the base strain for improving deinoxanthin production by optimizing the culture conditions. 

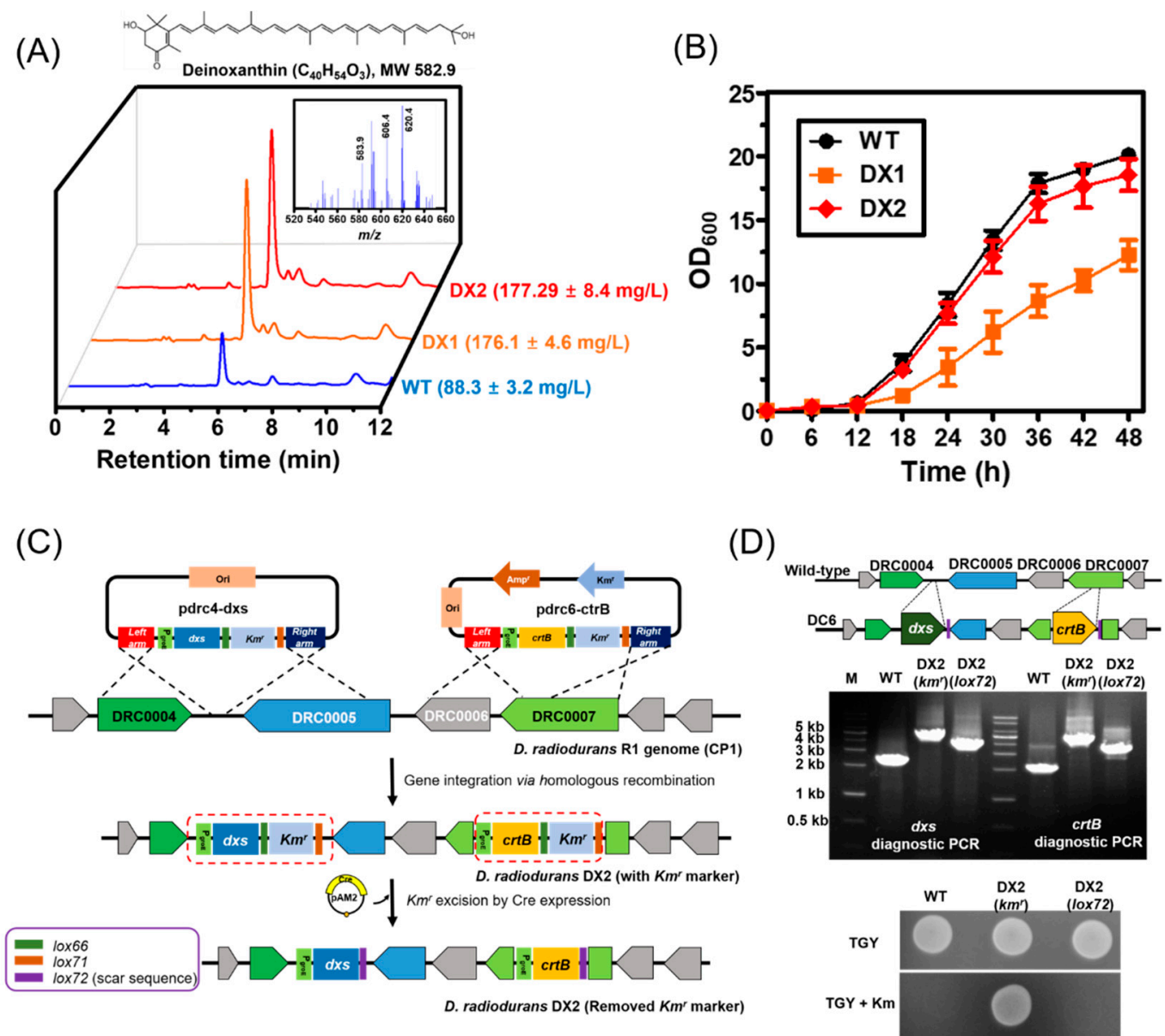

Figure 1. Cell growth and deinoxanthin production in engineered D. radiodurans strains. (A) Deinoxanthin production in the $D$. radidurans strains. HPLC spectra of the total carotenoids extracted from the $D$. radiodurans strains are presented. Inset presents the LC/MS spectra of total carotenoids obtained from the DX2 strain in positive ionization mode $\left(\mathrm{ESI}^{+}\right)$. The molecular ions at $m / z 583.9,606.4$, and 620.4 were assigned to $\left([\mathrm{M}+\mathrm{H}]^{+}\right),\left([\mathrm{M}+\mathrm{Na}]^{+}\right)$, and $\left([\mathrm{M}+\mathrm{K}]^{+}\right)$, which correspond to the presence of deinoxanthin. (B) Growth profiles of the plasmid-free D. radiodurans strain DX2 expressing the $d x s$ and $c r t B$ genes over $48 \mathrm{~h}$ of cultivation. (C) Scheme of the construction of plasmid- and antibiotic-free D. radiodurans DX2 strain for deinoxanthin overproduction. The detailed procedure is described in the Materials and Method section. (D) Diagnostic PCR of $d x s$ or $c r t B$ gene inserted in DX2 strain (upper panel). Plating assay of DX2 strain on trytone, glucose, and yeast (TGY) agar plate with or without kanamycin $(25 \mu \mathrm{g} / \mathrm{mL})$ (bottom panel). The data represent the mean and standard deviation of biological triplicates.

\subsection{Optimization of Culture Conditions to Enhance the Production of Deinoxanthin}

To increase deinoxantin production, the culture conditions were optimized, in particular the temperature and carbon source. In general, the groE promoter, which originates from the heat shock protein GroESL (an operon consisting of $d r 0606$ and dr0607) in D. radiodurans $\mathrm{R} 1$, is the most widely used promoter in $D$. radiodurans research due to its high stability and strong activity $[18,19]$. It is also known that the expression levels of genes regulated by the groE promoter vary depending on the temperature to which they were exposed [27]. Therefore, because $d x s$ and $c r t B$ were regulated by the groE promoter, it was reasoned that deinoxanthin production can be improved by optimizing the culture temperature. In order to determine the optimal temperature for deinoxanthin production, the mRNA expression levels of $g r o E, d x s$, and $c r t B$ were monitored in a temperature-dependent manner. As shown in Figure 2A, a gradual increase in mRNA expression patterns was confirmed as the temperature rose. A higher than 6-fold increase in the mRNA expression levels of groE was observed at $37{ }^{\circ} \mathrm{C}$ compared to those obtained at $30{ }^{\circ} \mathrm{C}$. With the use of the groE promoter at 
$37^{\circ} \mathrm{C}$, the mRNA expression levels of $d x s$ and $\operatorname{crtB}$ dramatically increased 13- and 18-fold, respectively, under the same conditions. Moreover, $256.5 \pm 13.8 \mathrm{mg} / \mathrm{L}$ of deinoxanthin was produced at $37^{\circ} \mathrm{C}$, which was approximately $32 \%$ higher than that obtained at 30 $(176.1 \pm 4.6 \mathrm{mg} / \mathrm{L})$ and $32{ }^{\circ} \mathrm{C}(174.4 \pm 7.3 \mathrm{mg} / \mathrm{L})$ (Figure $\left.2 \mathrm{~B}\right)$. These results suggest that external conditions that mimic the typical habitat of extremophilic microorganisms are an important consideration when engineering them for industrial applications.

(A)

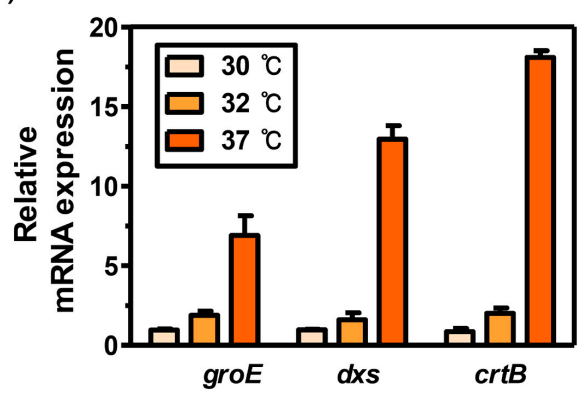

(C)

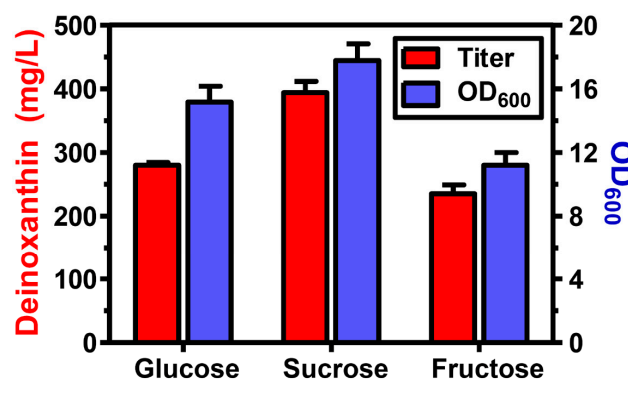

(B)
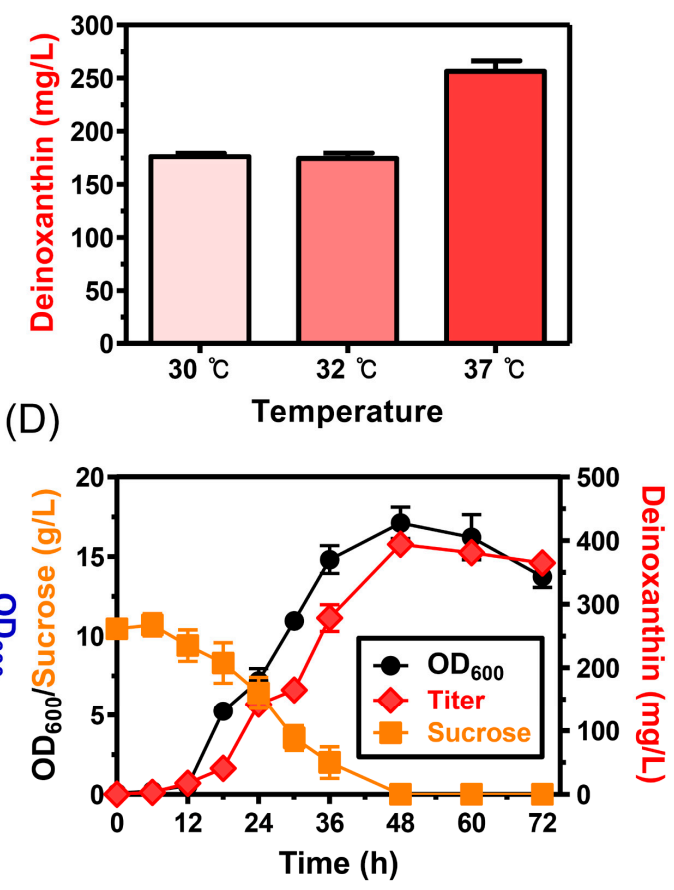

Figure 2. Deinoxanthin production by the D. radiodurans strain DX2 under different culture conditions. (A) Transcription profiles of the genes $g r o E, d x s$, and $c r t B$ based on the growth temperature. The strain DX2 cultured at $30^{\circ} \mathrm{C}$ was used as a control to normalize gene expression. Each sample was taken at $36 \mathrm{~h}$ for qRT-PCR analysis. (B) Deinoxanthin production under different growth temperatures. (C) Deinoxanthin production using different carbon sources $(10 \mathrm{~g} / \mathrm{L})$. (D) Shake flask batch culture in TY medium containing $10 \mathrm{~g} / \mathrm{L}$ sucrose under $72 \mathrm{~h}$ of incubation. All experiments were performed in triplicate, and the error bars represent the standard deviation.

Optimal carbon sources were also investigated. According to a previous report, sucrose, which consists of glucose and fructose, is the most effective carbon source for the production of carotenoids in tomato at the metabolic level [28]. To examine if this also applies to $D$. radiodurans, we tested sucrose, monomeric glucose, and monomeric fructose as the sole carbon sources for the production of deinoxanthin. As reported in the previous study, sucrose was found to be the optimal carbon source for deinoxanthin production. The strain DX2 supplemented with sucrose produced $394 \pm 17.6 \mathrm{mg} / \mathrm{L}$ of deinoxanthin, which was approximately $29.0 \%$ and $40.3 \%$ higher than that obtained from glucose $(280.2 \pm 4.3 \mathrm{mg} / \mathrm{L})$ and fructose $(235.3 \pm 1.9 \mathrm{mg} / \mathrm{L})$, respectively. These optimized conditions produced 102 $\pm 11.1 \mathrm{mg}$ deinoxanthin/g DCW with a yield and productivity of $40.4 \pm 1.2 \mathrm{mg} / \mathrm{g}$ sucrose and $8.4 \pm 0.2 \mathrm{mg} / \mathrm{L} / \mathrm{h}$, respectively, from $10 \mathrm{~g} / \mathrm{L}$ of sucrose (Figure 2C,D).

\subsection{Evaluation of the Antioxidant Activity of Deinoxanthin}

In order to investigate the industrial applicability and reliability of the deinoxanthin produced in the present study, in vitro DPPH (2, 2-diphenyl-1-picrylhydrazyl) radicalscavenging assays were performed. Figure 3A shows that deinoxanthin had significantly higher DPPH scavenging activity $(55.3 \pm 1.9 \%)$ than the carotenes phytoene $(25.5 \pm 3.2 \%)$, lycopene $(11.9 \pm 0.9 \%)$, and $\beta$-carotene (5.2 $\pm 1 \%)$. It also exhibited $21.7 \%$ higher DPPH 
radical-scavenging activity than that shown by astaxanthin ( $33.6 \pm 1.6 \%)$. In addition, the DPPH radical-scavenging activity of astaxanthin leveled off at $40 \%$ as the concentration increased, while that of deinoxanthin gradually increased in proportion to the concentration. A 2.4-fold increase in scavenging activity was also observed for $50 \mathrm{mg} / \mathrm{L}$ deinoxanthin compared to that observed for the same concentration of astaxanthin (Figure 3B).

(A)

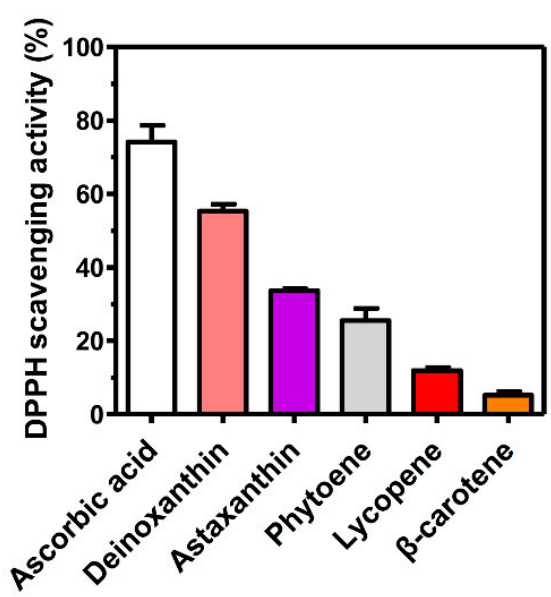

(B)

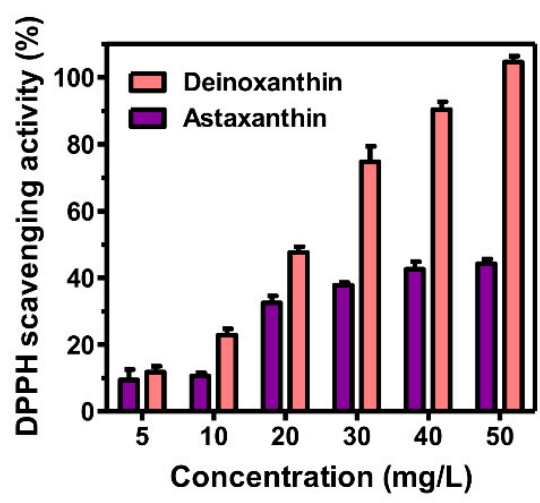

Figure 3. Results for 2, 2-diphenyl-1-picrylhydrazyl (DPPH) radical-scavenging assays. (A) Comparison of antioxidation activity with various carotenoids. Ascorbic acid (10 mg/L) was used as the positive control standard. All carotenoid concentrations were fixed at $20 \mathrm{mg} / \mathrm{L}$ for the DPPH assays. (B) DPPH scavenging activity of deinoxanthin and astaxanthin at different concentrations. All experiments were conducted in triplicate, and the error bars represent the standard deviation.

\section{Conclusions}

In this study, we reported the development of a genetically engineered strain of the extremophilic microorganism $D$. radiodurans that is capable of producing high levels of deinoxanthin following process optimization. Increased production of deinoxanthin was successfully achieved by integrating the genes $d x s$ and $c r t B$ into the $D$. radiodurans genome. Deinoxanthin production was enhanced further by comparing the mRNA expression levels of the genes involved in the rate-limiting steps. It was also proven that sucrose was the optimal carbon source for deinoxanthin production. The final engineered $D$. radiodurans DX2 was able to produce $394 \pm 17.6 \mathrm{mg} / \mathrm{L}(102 \pm 11.1 \mathrm{mg} / \mathrm{g}$ DCW $)$ of deinoxanthin from $10 \mathrm{~g} / \mathrm{L}$ of sucrose under optimal conditions. Furthermore, the feasibility of the microbial production of deinoxanthin was confirmed by in vitro DPPH radical-scavenging assays. In conclusion, the engineered strain and the strategies developed in the present study can be used as the basis for future industrial applications of extremophilic microorganisms.

Supplementary Materials: The following are available online at https:/ / www.mdpi.com/2076-260 7/9/1/44/s1, Figure S1: Schematic illustration of the deinoxanthin biosynthetic pathway and the metabolic engineering approach to enhancing deinoxanthin production in D. radiodurans; Figure S2. Verification of insertion of $d x s$ and $c r t B$ genes in D. radiodurans DX2 genome. (A) Diagnostic PCR of $d x s$ or $c r t B$ gene inserted in DX2 strain. (B) Plating assay of DX2 strain on TGY agar plate with or without kanamycin $(25 \mu \mathrm{g} / \mathrm{mL})$; Table S1: Primers used in this study.

Author Contributions: Conceptualization, S.-W.J. and Y.J.C.; methodology, S.-W.J., J.-H.K., J.-W.K., C.Y.K. and S.Y.K.; software, S.-W.J.; validation, S.-W.J., J.-H.K., J.-W.K. and C.Y.K.; formal analysis, S.-W.J., S.Y.K. and Y.J.C.; investigation, S.-W.J.; data curation, S.-W.J. and Y.J.C.; writing-original draft preparation, S.-W.J.; writing-review and editing, Y.J.C.; supervision, S.Y.K. and Y.J.C.; project administration, S.Y.K. and Y.J.C.; funding acquisition, S.Y.K. and Y.J.C. All authors have read and agreed to the published version of the manuscript.

Funding: This research was supported by the National Research Foundation of Korea (NRF) grant funded by the Korean government (MSIT) (2020R1A2C4001737) and the Korea Technology and 
Information Promotion Agency for SMEs (TIPA) grant funded by the Korean government (Ministry of SMEs and Startups) (no. S2954110).

Institutional Review Board Statement: Not applicable.

Informed Consent Statement: Not applicable.

Data Availability Statement: All data underlying the results are included as part of the published article and its Supplementary Materials.

Acknowledgments: This work was performed by the collaborative research project, named as "Deinoxanthins (Micrococcus ferment lysate)", with the LABIO biomaterial science research institute.

Conflicts of Interest: The authors declare no conflict of interest.

\section{References}

1. Young, A.J.; Lowe, G.L. Carotenoids-Antioxidant properties. Antioxidants 2018, 7, 28. [CrossRef] [PubMed]

2. Mordi, R.C.; Ademosun, O.T.; Ajanaku, C.O.; Olanrewanju, I.O.; Walton, J.C. Free radical mediated oxidative degradation of carotenes and xanthophylls. Molecules 2020, 25, 1038. [CrossRef] [PubMed]

3. Zaripheh, S.; Erdman, J.W. Factors that influence the bioavailability of xanthophylls. J. Nutr. 2020, 132, 531-534. [CrossRef] [PubMed]

4. Domínguez-Bocanegra, A.R.; Ponce-Noyola, T.; Torres-Muñoz, J.A. Astaxanthin production by Phaffia rhodozyma and Haematococcus pluvialis: A comparative study. Appl. Microbiol. Biotechnol. 2007, 75, 783-791. [CrossRef]

5. Asker, D.; Beppu, T.; Ueda, K. Zeaxanthinibacter enoshimensis gen. nov., sp. nov., a novel zeaxanthin-producing marine bacterium of the family Flavobacteriaceae, isolated from sea water off Enoshima island, Japan. Int. J. Sys. Evol. Microbiol. 2007, 57, 837-843. [CrossRef]

6. Saha, S.K.; Ermis, H.; Murray, P. Marine microalgae for potential lutein production. Appl. Sci. 2020, 10, 6457. [CrossRef]

7. Shindo, K.; Misawa, N. New and rare carotenoids isolated from marine bacteria and their antioxidant activities. Mar. Drugs 2014, 12, 1690-1698. [CrossRef]

8. Sugawara, T.; Ganesan, P.; Li, Z.; Manabe, Y.; Hirata, T. Siphoxanthin, a green algal carotenoid, as a novel functional compound. Mar. Drugs 2014, 12, 3660-3668. [CrossRef]

9. Takatani, N.; Nishida, K.; Sawabe, T.; Maoka, T.; Miyashita, K.; Hosokawa, M. Identification of a novel carotenoid, $2^{\prime}$ isopentenylsaproxanthin, by Jejuia palldilutea strain 11shimoA1 and its increased production under alkaline condition. Appl. Microbiol. Biotechnol. 2014, 98, 6633-6640. [CrossRef]

10. Mandelli, F.; Couger, M.B.; Paixão, D.A.A.; Machado, C.B.; Carnielli, C.M.; Aricetii, J.A.; Polikarpov, I.; Prade, R.; Caldana, C.; Paes Leme, A.F.; et al. Thermal adaptation strategies of the extremophile bacterium Thermus filiformis based on multi-omics analysis. Extremophiles 2017, 20,775-788. [CrossRef]

11. Lemee, L.; Peuchant, E.; Clerc, M.; Brunner, M.; Pfander, H. Deinoxanthin: A new carotenoid isolated from Deinococcus radiodurans. Tetrahedron 1997, 53, 919-926. [CrossRef]

12. Jeong, S.-W.; Kang, C.K.; Choi, Y.J. Metabolic engineering of Deinococcus radiodurans for the production of phytoene. J. Microbiol. Biotechnol. 2018, 28, 1691-1699. [CrossRef] [PubMed]

13. Kang, C.K.; Jeong, S.-W.; Yang, J.E.; Choi, Y.J. High-yield production of lycopene from corn steep liquor and glycerol using the metabolically engineered Deinococcus radiodurans R1 strain. J. Agric. Food Chem. 2020, 68, 5147-5153. [CrossRef] [PubMed]

14. Kang, C.K.; Yang, J.E.; Park, H.W.; Choi, Y.J. Enhanced lycopene production by UV-C irradiation in radiation-resistant Deinococcus radiodurans R1. J. Microbiol. Biotechnol. 2020, 30, 1-7. [CrossRef] [PubMed]

15. Tian, B.; Xu, Z.; Sun, Z.; Lin, J.; Hua, Y. Evaluation of the antioxidant effects of carotenoids from Deinococcus radiodurans through targeted mutagenesis, chemiluminescence, and DNA damage analyses. Biochim. Biophys. Acta Gen. Subj. 2007, 1770, 902-911. [CrossRef] [PubMed]

16. Tian, B.; Hua, Y. Carotenoid biosynthesis in extremophilic Deinococcus-Thermus bacteria. Trends Microbiol. 2010, 18, 512-520. [CrossRef]

17. Wang, C.; Zhao, S.; Shao, X.; Park, J.-B.; Jeong, S.-H.; Park, H.-J.; Kwak, W.-J.; Wei, G.; Kim, S.-W. Challenges and tackles in metabolic engineering for microbial production of carotenoids. Microb. Cell Fact. 2019, 18, 55. [CrossRef]

18. Airo, A.; Chan, S.L.; Martinez, Z.; Platt, M.O.; Trent, J.D. Heat shock and cold shock in Deinococcus radiodurans. Cell Biochem. Biophys. 2004, 40, 277-288. [CrossRef]

19. Meima, R.; Lidstrom, M.E. Characterization of the minimal replicon of a cryptic Deinococcus radiodurans SARK plasmid and development of versatile Escherchia coli-D. radiodurans shuttle vectors. Appl. Environ. Microbiol. 2000, 66, 3856-3867. [CrossRef]

20. Xu, Z.; Tian, B.; Sun, Z.; Lin, J.; Hua, Y. Identification and functional analysis of a phytoene desaturase gene from the extremely radioresistant bacterium Deinococcus radiodurans. Microbiology 2007, 153, 1642-1652. [CrossRef]

21. Zhang, L.; Yang, Q.; Luo, X.; Fang, C.; Zhang, Q.; Tang, Y. Knockout of crtB or crtI gene blocks the carotenoid biosynthesis pathway in Deinococcus radiodurans $\mathrm{R} 1$ and influences its resistance to oxidative DNA-damaging agents due to change of free radicals scavenging activity. Arch. Microbiol. 2007, 188, 411-419. [CrossRef] [PubMed] 
22. Zhou, Z.; Zhang, W.; Su, S.; Chen, M.; Lu, W.; Lin, M.; Molnár, I.; Xu, Y. CYP287A1 is a carotenoid 2- $\beta$-hydroxylase required for deinoxanthin biosysnthesis in Deinococcus radiodurans R1. Appl. Microbiol. Biotechnol. 2015, 99, 10539-10546. [CrossRef] [PubMed]

23. Tian, B.; Sun, Z.; Xu, Z.; Shen, S.; Wang, H.; Hua, Y. Carotenoid 3' $4^{\prime}$-desaturase is involved in carotenoid biosynthesis in the radioresistant bacterium Deinococcus radiodurans. Microbiology 2008, 154, 3697-3706. [CrossRef] [PubMed]

24. Choi, J.Y.; Lee, K.J.; Lee, P.C. Characterization of carotenoid biosynthesis in newly isolated Deinococcus sp. AJ005 and investigation of the effects of environmental conditions on cell growth and carotenoid biosynthesis. Mar. Drugs 2019, 17, 705. [CrossRef] [PubMed]

25. Jeong, S.-W.; Yang, J.E.; Im, S.H.; Choi, Y.J. Development of Cre-lox based multiple knockout system in Deinococcus radiodurans R1. Korean J. Chem. Eng. 2017, 34, 1728-1733. [CrossRef]

26. Chintong, S.; Phatvej, W.; Rerk-Am, U.; Waiprib, Y.; Klaypradit, W. In vitro antioxidant, antityrosinase, and cytotoxic activities of astaxanthin from shrimp waste. Antioxidant 2019, 8, 128. [CrossRef]

27. Schmid, A.K.; Lidstrom, M.E. Involvement of two putative alternative sigma factors in stress response of the radioresistant bacterium Deinococcus radiodurans. J. Bacteriol. 2002, 184, 6182-6189. [CrossRef]

28. Télef, N.; Stammitti-Bert, L.; Mortain-Bertrand, A.; Maucourt, M.; Carde, J.P.; Rolin, D.; Gallusci, P. Sucrose deficiency delays lycopene accumulation in tomato fruit pericarp disc. Plant Mol. Biol. 2006, 62, 453-469. [CrossRef] 